\title{
DEVELOPMENT OF A NOVEL SNOM PROBE FOR IN LIQUID BIOLOGICAL SAMPLES
}

\author{
F. ARMANI, A. BOSCOLO, M. BRESSANUTTI, M. DALLE FESTE, \\ B. PIUZZI, A. DE VECCHI, E. VIVIANI
}

Dept. of Engineering and Architecture, Trieste University, APL laboratory, Trieste, Italy, boscolo@units.it

\author{
M. ZWEYER
}

Clinical Dept. of Medical, Surgical and Health Sciences, Trieste University, Trieste, Italy

This work is focused on the study and implementation of a novel method for the development of probes for Scanning Near-field Optical Microscopy (SNOM). The proposed approach is based on the mechanical impedance matching between the optical fiber tip and the resonating tuning fork. This methodology allowed an increase of the quality factor of the piezoelectric resonator used as atomic force transducer in the SNOM probe, thus increasing its overall sensitivity. This kind of probes are often used on biological soft samples in liquid. The presence of water medium has a strong dumping effect on probe sensitivity. Experimental validation of the proposed methodology showed an increase of robustness of SNOM probes also for in liquid samples.

\section{Introduction}

Scanning Near Field Optical Microscopy (SNOM) is a branch of scanning probe techniques, which is based on the double interaction atomic force and lighttunneling effect. SNOM is gaining interest among other well consolidated techniques as SEM, AFM and TEM [1]. In particular SNOM is characterized by its unique capability to detect structures, through the near-field optical interaction up to $50 \mathrm{~nm}$ and by a low impact on the specimen, comparable or even better than standard AFM techniques [1,2]. SNOM microscopy can be used with soft samples as it is characterized by low complexity in preparing samples, is less invasive and shows higher surface topographic resolution respect to conventional optical and confocal microscopes.

\begin{tabular}{ccccc} 
& \multicolumn{4}{c}{ Quality Factor } \\
\cline { 2 - 5 } Fiber layout & Packaged Quartz & Opened Quartz & $\begin{array}{c}\text { Quartz with } \\
\text { fiber, in air }\end{array}$ & $\begin{array}{c}\text { Quartz with } \\
\text { fiber, in water }\end{array}$ \\
\hline Standard & 20000 & 15000 & 3000 & 500 \\
\hline APL Layout & 20000 & 15000 & 4500 & 1800
\end{tabular}

Table 1: performance comparison of SNOM probes built with different optimizations 
A SNOM probe is constituted of a primary and a secondary transducer. An optical fiber tip allows the near field interaction with the specimen while a second transducer exploits some electro-mechanical interaction to detect the weak atomic forces that occur between the tip and the surface [2].

As other emerging technologies, SNOM still presents issues related to imaging repeatability of in liquid samples. That is, the $\mathrm{Q}$ value of the probe, which is an important indicator of the sensitivity performance, abruptly decrease when the fiber is dived in the liquid cell $[3,4]$.

The performance enhancement of SNOM probes has been achieved through the study and complex activity of analysis, modeling and development of secondary transducers and related electronics, to meet the requirements of accuracy and repeatability necessary to bring in liquid SNOM microscopy to a high level of confidence.

\section{Optimization}

To optimize the SNOM probe, an electromechanical model of the resonator has been implemented on a commercial multiphysics finite element simulator (Comsol Multiphysics) which has been tuned using experimental data. This had permitted the identification of the zero-strain axis of the tuning fork, the zone with less strain during arm oscillation, and thereby determine the bonding area in which the resonator is less susceptible to fiber gluing with a consequential improvement of the $\mathrm{Q}$ factor (Table 1).

The SNOM probe has been characterized at different conditions of immersion in water and optical fiber layout using a HP4192A commercial electrical impedance analyzer. Secondly it has been mounted on a commercial SNOM microscope (TriA-SNOM by A.P.E. Research) and characterized as a force transducer to assess its sensitivity both in air and in water.

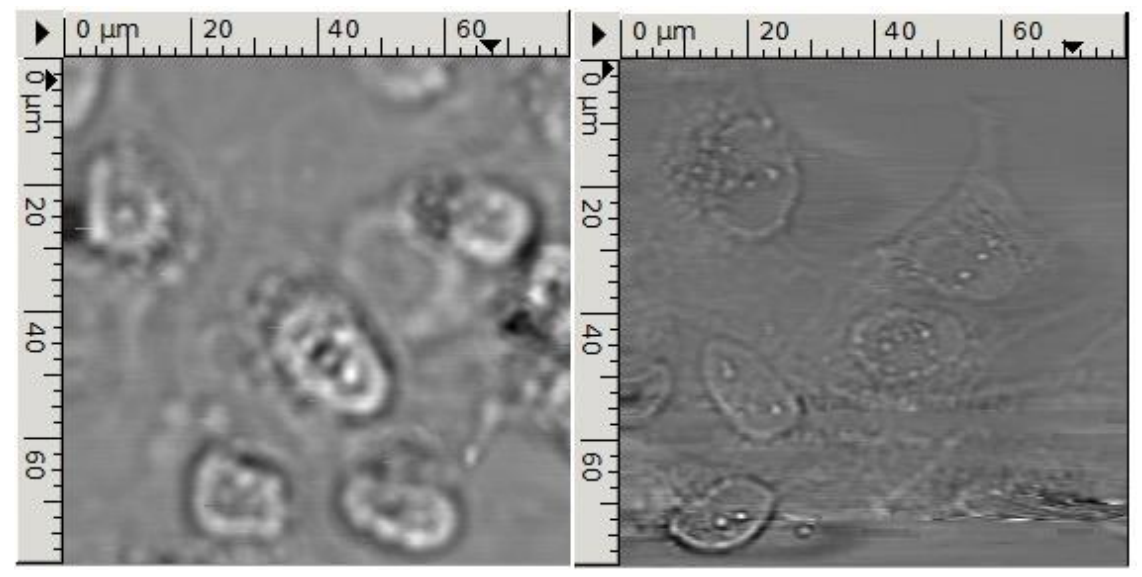

Figure 1: Reflective near field (left) and transmission far field (right) of a gently bonded mesothelium in physiological solution 


\section{Characterization}

The probe has then been tested with different samples as a brass plate, a strong fixed cell sample and a soft fixed one. Topographic, transmission and reflective optical images have been acquired (Figure 1). SNOM probes metrological characterization was then performed using as reference a certified secondary standard. This was a Micromasch TGZ02, a silicon grating used for the characterization of SPM systems. Results (Figure 2, 3) has confirmed that the resolution of this probe is compatible with SPM probe standards; moreover it can perform with similar sensitivity both in air and in liquid.

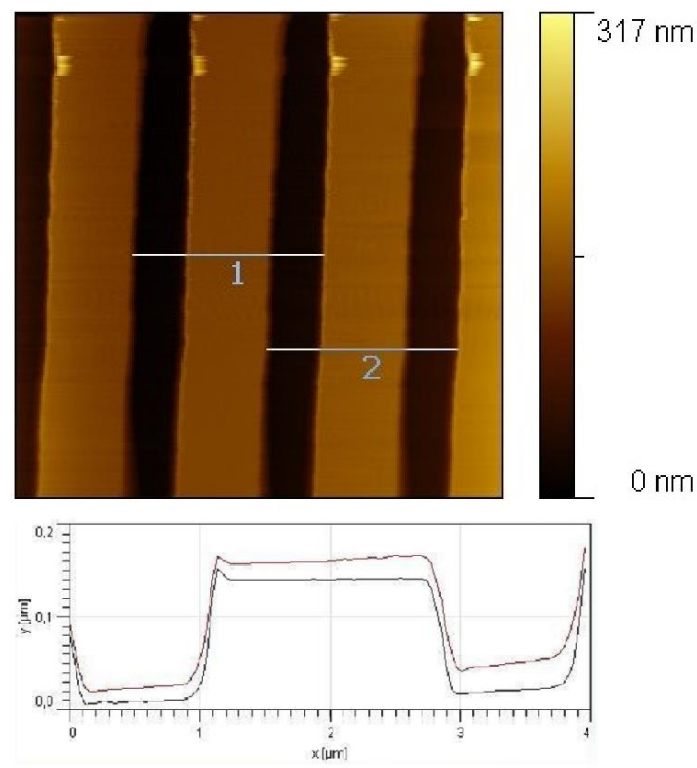

Figure 2: topographic image of a TGZ02 grating standard (top) and related depth profile (bottom) 


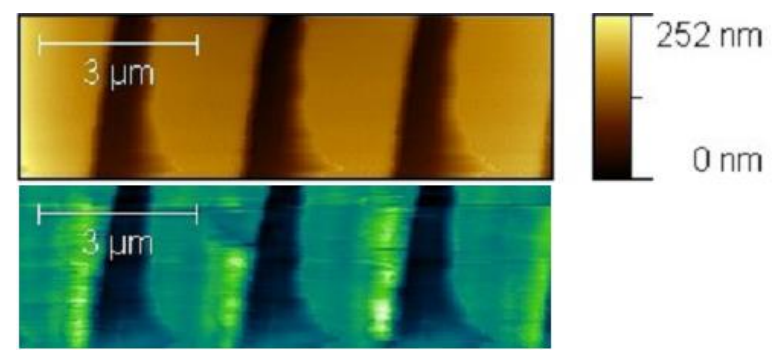

Figure 3: TGZ02 grating standard in water images: topographical (top) and optical (bottom). This was realized in near field reflective mode with a $532 \mathrm{~nm}$ laser source

\section{Conclusions}

The proposed work showed that an in depth physical modeling of SNOM probes is essential to optimize their performance. The use of a typical electronic approach, such as impedance matching, has been found to be very effective also when applied in a mechanical context. The use of the quality factor as an indicator of probe sensitivity is a good starting point for probes performance optimization.

The metrological characterization confirmed an improvement of probe sensitivity on a grating standard, both in air and in water. This increase in sensitivity is a promising achievement for the acquisition of high quality SNOM images for in liquid biological samples and gently fixed samples, which are usually destroyed by conventional scanning probes.

\section{Acknowledgments}

This work was supported by art. 23 LR 26/2005 Regione FVG, as part of the project: "Development of SNOM microscopy as an emerging technique in the biomedical sciences" (2010-2011).

\section{References}

[1] M. Zweyer, B. Troian, V. Spreafico, S. Prato, "SNOM on cell thin sections: Observation of Jurkat and MDAMB453 cells", Journal of Microscopy, 229, pp. 440-446, 2008.

[2] R. Brunner, A. Bietsch, O. Hollricher, O. Marti, "Distance control in near-field optical microscopy with piezoelectrical shear-force detection suitable for imaging in liquids", Review of Scientific Instruments, 68 (4), pp. 1769-1772,1997.

[3] W.H. Rensen, N.F. Van Hulst, A.G. Ruiter, P.E. West, "Atomic steps with tuning-fork-based noncontact atomic force microscopy", Applied Physics Letters,75, pp. 1640-1642, 1990.

[4] F.J. Giessibl, "Atomic resolution on $\mathrm{Si}(111)-(7 \times 7)$ by noncontact atomic force microscopy with a force sensor based on a quartz tuning fork", Applied Physics Letters, 76 (11), pp. 14701472,2000 